\title{
Endocrinology and Metabolic Disorders
}

\author{
Precocious puberty
}

\section{Soheilipour $\boldsymbol{F}$ \\ Iran University of Medical Sciences, Tehran, Iran}

Precocious puberty refers to the appearance of physical and hormonal signs of pubertal development at an earlier age than is considered normal. The onset of puberty before 7 or 8 years for white girls and 9 years for boys is considered precocious. Early onset of puberty can cause several problems. The early growth spurt initially can cause tall stature, but rapid bone maturation can cause linear growth to cease too early and can result in short adult stature. The early appearance of breasts or menses in girls and increased libido in boys can cause emotional distress for some children. Premature pubarche and premature thelarche are 2 common, benign, normal variant conditions that can resemble precocious puberty but are nonprogressive or very slowly progressive. A thorough history, physical examination, and growth curve review can help distinguish these normal variants from true sexual precocity. If the history, physical examination, and laboratory data suggest that a child exhibits early and sustained evidence of pubertal maturation, the clinician must differentiate central precocious puberty (CPP) from precocious pseudo puberty. Central precocious puberty, which is gonadotropindependent, is the early maturation of the entire hypothalamic-pituitary-gonadal (HPG) axis, with the full spectrum of physical and hormonal changes of puberty. Precocious pseudo puberty is much less common and refers to conditions in which increased production of sex steroids is gonadotropin-independent (Precocious pseudopuberty). Correct diagnosis of the etiology of sexual precocity is critical, because evaluation and diagnosis of patients with precocious pseudopuberty is quite different than that for patients with central precocious puberty.

Keywords: Precocious Puberty, Central, Pseudo Puberty, Diagnosis, Treatment

\section{The prevalence of diabetes in students arriving to school in Nahavand city in 1392-93}

\section{Mortazavi SS}

Hamedan University of Medical Sciences, Iran

Background: Diabetes is a metabolic complex disease in which such factors as intrinsic aptitude, environmental conditions like life style, weight, food patterns range and people's awareness have determining role in it's prevalence. This chronic disease is classified into 2 types.

Methods: This investigation was carried out through medical examining in the health measurement plane of the pre-elementary students (7 years) in Nahavand city, 20122013. Data was gathered through interview and examining; and it was analyzed through statistical testing of Chisquare, analysis and variance, $t$ test, and coefficient correlation. $\mathrm{P}<0.05$ was considered as a meaningful level. Findings: 2223 students (1125 boys, 1098 girls) were investigated regarding diabetes signs from which 420 people were considered as patients. Out of this number, 27 people were confirmed to have diabetes ( 12 boys, 15 girls).
There was not a meaningful differences between sex and the disease spread. 19 patients of 27 patients were informed of their own disease. A meaningful relationship was observed between the parents' education and the disease prevalence. A meaningful relationship was observed between the parents' education and awareness of the child disease. A significant relationship was not observed between the parents' age and the diabetes prevalence. Conclusion: This study and similar research indicate that with increasing growth of the number of people involved with diabetes 1 (students are treated as a branch of this group) the necessity of early diagnosis and educational programs regarding diabetes are unavoidable in schools. Thus, it is suggested that essential teaching for teachers and tutors concerning the students affected with diabetes will improve educational achievement of the students.

Keywords: Prevalence, Diabetes, Student

\section{The diagnostic dilemmas of skeletal dysplasia: classification, incidence and mode of inheritance of different type (a clinical and radiological overview)}

\section{Mearadji $M$ \\ Erasmus MC, Sophia Childrens Hospital, Rotterdam, the Netherlands}

Skeletal dysplasia are a primary result of mutation genes that are expressed in chondro-osseous tissue. However, there are also some other forms of secondary dysplasia caused by abnormalities of extra-osseous factors with effects on the skeletal system like metabolic errors in hypophosphatemic rickets. International nosology and classification of genetic disorders of bone (published 2009) includes 37 groups; from 250 well defined disorders about 70 are often lethal in the perinatal period. Primarily the classification of skeletal dysplasia is based on radiological features of skeletal changes. However, the progress in molecular genetics especially in the last decade permit owing to distinctions at the DNA level refinements in classification resulting in an increasing number and subtypes of dysplasias. The similarity of morphological findings with or without the same inheritance as well as their rare incidence are all reasons for diagnostic dilemma in this field. The first step in the diagnosis of skeletal dysplasia is to observe a disproportionate skeletal development, unusual habitués with or without mental retardation as well as other clinical signs. The second step is more difficult and includes genetic counseling and interpretation of radiologic skeletal changes in affected patients and their families. The rare incidence of skeletal dysplasia on one hand and its morphologic diversities on the other hand require some experience and therefore, centralization of knowhow in this field is recommendable. In this manner medical geneticists and radiologists gain the ability and expertise in this field. The aim of this presentation is to give an overview of skeletal dysplasia; a large number of different types will be shown. Special attention should be paid to their inheritance, molecular pathology and incidence as well as the radiological findings. 
Keywords: Skeletal Dysplasia, Classification, Inheritance, Radiology

\section{Effect of growth hormone deficiency on brain MRI findings among children with growth restrictions}

Rajabi Eslami S, Naderi F, Sohrab Afshari Mirak S, Khak M, Amiri J, Beyrami B, Shekarchi B, Poureisa M Qom University of Medical Sciences, Iran

Background: Growth hormone deficiency (GHD) is a major problem among children with short stature. In this study, the role of brain magnetic resonance imaging (MRI) in defining the underlying defects among short children with GHD was evaluated.

Methods: In a cross-sectional study, data of 158 children were evaluated. Growth hormone (GH) levels were measured using stimulating tests and brain MRI with gadolinium contrast was applied, as well.

Findings: Some $25.3 \%$ of patients had GHD with a mean age of 8.01 \pm 3.40 years. MRI results showed 35 patients as normal, four patients with pituitary hypoplasia, and one patient with microadenoma. The MRI results were significantly associated with GH levels and presence of other endocrine disorders. There was a significant association between prenatal disorders and patients' bone age delay.

Conclusion: In patients with severe GHD and patients with multiple pituitary hormone deficiencies, MRI is more likely to be abnormal, and bone age is much delayed in patients with history of prenatal disorders.

Keywords: Bone Age, Growth Hormone Deficiency, Magnetic Resonance Imaging, Short Stature

\section{Pediatric metabolic bone diseases classification and an overview of clinical and radiological findings}

\section{Mearadji $M$ \\ Erasmus MC, Sophia Childrens Hospital, Rotterdam, the Netherlands}

From a practical point of view the metabolic bone diseases should be categorized etiologically because of an overlap of some endocrine bone diseases and skeletal dysplasia. The metabolic bone diseases affecting bone formation and mineralization may be classified as follows (4 groups): A. Disorders with insufficient mineralization of the organic matrix: The group includes different types of rickets following vitamin D abnormalities. The most frequent causes of vitamin D deficiency are nutritional, hepatic- and renal disorders. Also rickets of prematurity, renal tubular disease and hypophosphatemia should be classified in this group. Phosphopenic rickets is an autosomal dominant abnormality with phosphate wastage. The radiological findings in all different types of rickets are similar with some differences depending on severity and according to type with variable structural changes. B. Abnormalities of bone matrix formation: Osteogenesis imperfecta with decreased bone mineralization due to insufficient matrix formation and quantitative and qualitative defects in synthesis of type I collagen. The radiographic feature has a wide variation in 5 major types and subtypes. In general osteoporosis, retarded calvarial bone formation, rib- and tubular bone fractures and collapsed vertebral bodies are all characteristic findings as well as deformities of limbs. Menkes disease and scurvy: collagen synthesis requires ascorbine acid and copper dependant ascorbine acid oxidase. Therefore children with scurvy and Menkes disease (copper deficiency syndrome) are both affected in addition to other clinical signs and symptoms with skeletal changes as a result of osteoid deficiency. The characteristic changes are osteopenia, bone fragility, metaphyseal fractures and others. Abnormalities of increased or decreased bone resorption: The major cause of increased bone resorption is hyperparathyroidism in children with renal failure. Radiolocigally they manifest themselves as renal osteodystrophy with subperiostal resorption along the phalanges, involving also the distal phalangeal tufts. Soft tissue calcification and fractures among others also occur. Hyperphosphatasia is a rare autosomal recessive disease and is also a condition with increased bone turnover, and elevated serum alkaline phosphatase level. It appears to be due to deficiency of osteoprotegerin. Radiological signs are expanded and bowed diaphyses. There is a marked demineralization with a heterogeneous pattern and cortical thickening, and pathological fractures. Osteopetrosis is an example of decreased bone resorption with calcified cartilage and abnormality of the osteoclast function. The different types are divided into infantile and juvenile. The juvenile type is categorized in six sub groups. Mostly they are autosomal recessive herited with exception of autosomal dominant type I and II of the juvenile type. D. Pharmacologic and toxic changes in the skeleton. There are several pharmacologic and toxic materials affecting the resorption of bone and calcified cartilage, including side effects of biphosphanate and prostaglandine and toxic effects by hypervitaminosis A and D and heavy metal poisoning (lead). Also primary oxalosis due to oxalate overproduction should be classified in this group. Obvious radiologic changes are different and characteristic. E.g. increased bone density and opaque dense lines due to cyclic therapy will be found following biphosphanate treatment. Lead lines due to excessive calcified cartilage are observed in lead poisoning. Based on the above mentioned data, numerous types of metabolic bone disorders will be shown with special attention for etiology, typical structural and anatomic changes in different disorders.

Keywords: Pediatric, Metabolic, Bone, Classification, Clinical, Radiology

\section{Diabetic ketoacidosis and predisposing factors in diabetic patients}

\section{Alijanpour M, Shabanzadeh Z, Rezapour M, Bijani A, Aghajanpour $\boldsymbol{F}$ \\ Babol University of Medical Sciences, Iran}

Background: Diabetic ketoacidosis is a final result of severe insulin deficiency in type 1 diabetes that occurs in $20-40 \%$ of children and it is a life-threatening complication. This phenomenon is a medical emergency and requires prompt diagnosis and treatment. The aim of this study was investigation of frequency and causes of diabetic ketoacidosis and its consequences to prevent complications.

Methods: This study was performed on 128 diabetic patients in Amirkola Children's Hospital (1384-1392). Patient information was recorded in a predetermined questionnaire and data were then entered into SPSS version 
18.0 software for statistical analysis.

Findings: Of 128 diabetic patients hospitalized in Amirkola Children's Hospital, $54.7 \%$ of patients were male and $45.3 \%$ were female with an average age of $8.57 \pm 3.27$ years. $103(80.5 \%)$ patients were referred to this hospital for the first time and did not have a history of diabetes and $25(19.5 \%)$ patients were previously treated with insulin. Of total 128 patients, $71(55.5 \%)$ had some degree of ketoacidosis; severe ketoacidosis was the most common with $28.1 \%$. Among patients with a diagnosis of ketoacidosis (71 patients), 54 patients (76\%) presented with diabetic ketoacidosis as the first symptom of diabetes. In general, the predisposing factors of ketoacidosis was seen in $38 \%$ of patients, respiratory infections $24.3 \%$, urinary tract infections $2.8 \%$ and gastrointestinal infections $4.30 \%$. In patients with previous diagnosis of diabetes mellitus and admission with diabetic ketoacidosis, 52.9\% of them had previous treatment cessation. Complications during treatment of ketoacidosis in patients like hypokalemia $21.2 \%$, hyperkalemia $15.4 \%$, hyponatremia 28.1 and hypernatremia $11.2 \%$ was observed.

Conclusion: Based on the results of this study, high prevalence of ketoacidosis in our patients than any of the other studies, seem to be related to lack of informations about diabetes mellitus between population and medical field that require to universal education.

Keywords: Diabetes, ketoacidosis, brain edema, hyponatremia, hyperkalemia

\section{Partial empty sella syndrome : a case of short stature}

\section{Noorian $S$ \\ Department of Pediatrics, Bahonar Hospital, Alborz University of Medical Sciences, Karaj, Iran}

Background: When the pituitary gland is not seen within the sella turcica on radiologic imaging studies, is called empty sella syndrome. It's an infrequent finding in childhood, but is more frequently seen in girls than boys. Pituitary hormones deficiency is present in less than $10 \%$ of such cases. The single most common endocrine abnormalities noted in children with primary empty sella is growth hormone deficiency.

Case presentation: An 11.5-year-old girl was referred for evaluation of short stature. Her weight was $22 \mathrm{~kg}$ and height $128 \mathrm{~cm}$, which was $-3.6 \mathrm{SD}$ below the mean. She had also not any signs of breast development and puberty at this age. After ruling out systemic illness, the patient underwent specialized tests and radiologic evaluation. She was diagnosed to have $\mathrm{GH}$ deficiency by poor response to $\mathrm{GH}$ stimulation test, other endocrine evaluation revealed that LH, FSH and estradiol levels were prepubertal, and the rest pituitary hormone were all normal. MRI showed a partial empty sella on pituitary magnetic resonance imaging. On the above findings diagnosis of partial empty sella syndrome was made.

Conclusion: Empty sella is sometimes associated with pituitary hypofunction and is most often discovered during imaging studies. MRI or CT scans are useful in differentiating empty sella from other etiologies of pathologic short stature. Treatment involves replacing the hormones that are lacking.

Keywords: Empty Sella, Short Stature, Pituitary Hypofunction

\section{Evaluation of the patients with Diabetes Mellitus in ediatric hospital of Khoramabad}

\section{Mohsenzadeh N, Mohsenzadeh A \\ Lorestan University of Medical Siences, Khoramabad, Iran}

Background: Diabetic Mellitus is the most commen endocerin-metabolic disorder of childhood and adolescence with important consequences for physical and emotional development. Mortality and morbidity of acut metabolic derangments and long term complications that affect small and large vessells result in retinopathy, nephropathy, ischemic heart disease and arterial obstruction with gangrene of the extremities. The aim of this study was evaluation of patients with diabetic mellitus in pediatric Hospital of Khoramabad in 2009-2013.

Methods: This study was done as a cross-sectional. Sampeling was simple. All the patiets that hospitalized with diagnosis of diabetic mellitus were evaluated. The studied variables included age, sex, clinical and paraclinical sign, duration of hospitalization and treatment. The data was collected by questionaire and analyzed by SPSS software. Findings: Out of 100 patients, $70 \%$ were females and $30 \%$ were males. $10 \%$ were $<5$ years old, $50 \%$ were $5-10$ years old and $40 \%$ were $>10$ years old. $55 \%$ were urban and $45 \%$ were rural. $35 \%$ had polydepsia, polyuria and polyphagia. $70 \%$ were hospitalised with DKA. 25\% had nocturnal enuresis and constipation. $20 \%$ had an infection disease. Duration of disease onset in 65\% was 15-30 days. Mean of the BS was $384 \pm 96.75 \%$ had glucosuria $3+$. Duration of hospitalization in $70 \%$ was 6-9 days. One of the patients diad.

Conclusion: In this study $70 \%$ of the patients were females. $70 \%$ were hospitalised with DKA. Considering the diabets is the most common endocerine disorder, we suggest educational programs for parents.

Keywords: Diabetic Mellitus, DKA, Children

\section{Genetics \& public health: genetic prevention in the PKU program of Iran}

\author{
Hajivalizadeh F, Azimi S, Kazemi P, Ehsaninejad S, \\ Dormiani N, Ziadloo T, Vahidi M \\ Ministry of Health and Medical Education, Iran
}

Background: A program for prevention and control of Hyperphenylalaninemia as the head part of a comprehensive programme for hereditary metabolic disorders was initiated by the ministry of health in 1997. The program mainly aim to provide patients with standardized care and their family and relatives with prevention services.

Methods: Thirty five designated children hospitals in the provinces are responsible to register PKU patients of the province. A clinical team including a metabolic-endocrine subspecialist (or general pediatrician), a nutritionist, a psychiatrist, welfare worker, lab expert and drug technician, work together to deliver services in the regular bases. Parents are referred to the genetic counseling services of the district by the clinician of the team. When patients screened receive their services. Parents in the sessions are informed of the risks and options to avoid them. Patient's relatives who are at risk of the diseases are also invited in following sessions and receive genetic counseling services. 
Findings: Each year 1500000 newborn are screened at the national level. Until 2012, 1599 patients registered in the 35 hospitals of provinces. Fifty seven present of the families referred to the genetic counseling attend the sessions and genetic files were produced for them among which 534 families has done the prenatal diagnosis tests. Seventy fetuses were found affected and from which 68 parents chose to abort the affected fetuses after post test genetic counseling.

Conclusion: In the national PKU prevention and control program of Iran, care and prevention are integrated in the public health and people are provided with the clinical and preventive services. In the context of this program preventive genetic services are accessible equitably with consideration to autonomy of the parents and patients and other important ethical issues of providing services.

Keywords: Genetic counseling, PKU, newborn screening

\section{Prevalence of vitamin D deficiency in healthy children in Kashan}

Tabatabaei $F^{1}$, Sharif $M^{2}$, Madani $M^{3}$, Tabatabaee $Z^{4}$

${ }^{1}$ Isfahan Endocrine and Metabolism Research Center, Isfahan University of Medical Sciences. ${ }^{2}$ Trauma Research Center. ${ }^{3}$ Student Research Committee. ${ }^{4}$ Department of Clinical Psychology, Kashan University of Medical Sciences, Iran

Background: The data on the prevalence of vitamin D deficiency in healthy children from Iran is limited. Therefore, the current study was performed to evaluate the level of vitamin D in healthy children in Kashan.

Methods: The cases was selected from the healthy children aged 1-6 years who referred to the health centers for checking their weight and height during 2013. The children who were suffering from liver, kidney or any endocrine diseases or were on supplementary diet for vitamin D were excluded from the study. Prior to the start of the protocol, 3 cc of venous blood was drawn from the children. Standard DIA source kit and ELISA method were used to measure the level of 25-hydroxi vitamin D. Based on the criterion set results of the recent studies, the levels of vitamin D below the 10 nanograms per milliliter is classified as severely low, between 10 to 30 as low, 30 to 100 as normal and above 100 is defined as the toxic level.

Findings: In total, 100 children (54 boys, 46 girls) with mean age of 2.6 years were enrolled. The percentages of children with severely low, low, normal and toxic levels of $25(\mathrm{OH}) \mathrm{D}$ were $32,47,21$ and 0 , respectively.

Conclusion: Level of vitamin D was remarkably low in the children in this study. Nevertheless, further studies are recommended in this regard.

Keywords: Vitamin D Deficiency, Healthy Children, Kashan

\section{Glutaric aciduria Type II, clinical presentation and medical approach}

\section{Mosallanejad A, Abbasi F, Setudeh A \\ Shahid Beheshti University of Medical Sciences, Iran}

Background: Glutaricaciduria II is a rare autosomal recessive disorder. This results from mutation in at least 3 genes included ETFA, ETFB, and ETFDH.

Case presentation: The patient, a boy, was the first child of consanguineous healthy parents. He was refered for seizure. On examination, he had no dysmorphic feature. In the lab tests, he had hypoglycemia, metabolic acidosis without ketosis. Blood spot screening from the baby by tandem mass spectrometry showed increases in long chain and medium chain acyl carnitines. Gas-chromatography mass spectrometry revealed significant elevation of urinary glutaric acid, 2 Hydroxyglutaric acid and Isobuteric acid. The pattern accumulated metabolites was consistent with defect in activity of acyl-coA dehydrogenase. He was treated with riboflavin and carnitin. Finally, he developed liver function impairment and lactic acidosis. Then, he died at 5 month of age. The genetic analysis confirmed glutaricaciduria II, [mutation in the ETFDH gene G>c(p.Gly381 Arg)c,1141].

Conclusion: Glutaricaciduria II ,or multiple acyl-coA dehydrogenase (MADD), is a rare autosomal recessively inherited disorder of fatty acid, amino acid and choline metabolism. The disorder is due to a defect in either the alfa or beta-subunit of electron transfer flavoprotein (ETFA ,ETFB, and ETFDH). Patient with this disorder have an unusual pattern in which a large number of organic acids are found in elevated amounts in plasma and urine. Lactic acid and glutaric acid are the most prominent. Some patients respond dramatically to pharmacological doses of riboflavin and carnitin.

Keywords: Glutaric Aciduria Type II, Multiple Acyl-Coa Ehydrogenase Deficiency

\section{New medical approach to diabetes mellitus}

\section{Zaridoost A}

Gilan University of Medical Sciences, Iran

Regarding worldwide accelerating high incidence of diabetes mellitus, new hopeful medical horizons are appearing every day. Drug discovery and development to approval is a long risky road, about $10-15$ years. For every 5000-10000 compounds in pipelines only one substance will be approved. Variety of hypoglycemic agents was presented to diabetic patients. Generally they were divided to 7 groups: -Insulins: ultrashort acting, short acting, intermediate acing, long acting. -Insulin sensitizers acting through liver: Biguanides. -Insulin sensitizers acting through peripheral tissues: thiazolidinediones. -Insulin secretagogues: sulfonylureas and meglitinids. Carbohydrate absorption inhibitors: $\alpha$-glucosidase inhibitors. -Incretin mimetics, affect as glucagon like peptide 1(GLP-1) or inhibit dipeptidyl peptidase-IV (DPPIV), and Amylinomimetics that promote satiety and reduce appetite and rate of gastric emptying. The last group of medication which has been approved is sodium glucose cotransporter2 (SGLT-2) inhibitors which inhibit glucose reabsorption in kidneys. Canagliflozin and Dapagliflozin are two FDA approved medicines of them. They are applicable for type two diabetics with appropriate kidney function (GFR > $60 \mathrm{ml} / \mathrm{min} / 1.75 \mathrm{~m}^{2}$ ). Inhaled insulin were collected from markets in 2007 in the USA, recently is produced in a safe and more convenient form. It is prepared in single dose cartridges and the delivery system is a whistle size device. Both the insulin and the powder are nearly completely cleared from the lungs of healthy individuals within 12 hours of inhalation; only 0.3 percent of the insulin and 0.4 percent of the powder concentration remain after 12 hours. Ultra-long acting Insulin (degludec) is multihexamer insulin, mixable with short acting insulins, activated in physiologic PH subcutaneously once a day or three times a 
week. Artificial pancreas is composed from injecting insulin pomp and a blood glucose monitoring device that inject appropriate dosage of insulin regarding blood glucose. It stops insulin infusion when blood glucose is in critical range.

Keywords: Diabetes Mellitus, Insulins, Inhaled Insulin, New Approach, Artificial Pancreas

\section{Physical activity and exercise in children with diabetes mellitus type 1}

\author{
Sayarifard $F^{1}$, Sayarifard $A^{2}$, Yaghmaie $B^{2}$ \\ ${ }^{1}$ Growth and Development Research Center. ${ }^{2}$ Children's Medical \\ Center, Tehran University of Medical Sciences, Iran
}

Doing exercise is one of the important components in diabetic children management. ADA recommended that all children with diabetes should be encouraged to do exercise at least 60 min of physical activity each day. The benefits of exercise are weight control, reduced cardiovascular risk, reduced $\mathrm{Hb} \mathrm{A} 1 \mathrm{C}$, improved insulin sensitivity and improved sense of well-being. Due to increased insulin sensitivity, risk of post-exercise nocturnal hypoglycemia is high, if bedtime blood glucose level is $<125 \mathrm{mg} / \mathrm{dL}$. Since hypoglycemia is common during the exercise, shortly after, or even up to 24 hours afterwards, these recommendations for diabetic patients which are approved by ISPAD and ADA must be taken into consideration: - Adjusting insulin regimen to activity - Reducing insulin dosage before exercise if necessary. - Discussing type and amount of carbohydrate required for specific activities - Having snacks and sugar in case of emergency - Avoiding any exercise if pre-exercise blood glucose levels are high $(>250$ $\mathrm{mg} / \mathrm{dl}$ ) with ketonuria/ketonemia. Pre -exercise Blood glucose between 100 up to $250 \mathrm{mg} / \mathrm{dl}$ is safe. • Drinking a lot of sugar-free fluids during exercise. - Not having injected insulin at a site that will be heavily involved during activity. The children with diabetes should be allowed to choose their favorite sports. Diabetes does not limit their ability to do exercise. They should record details of their activity, insulin, food and glucose results for good diabetes control during exercise.

Abbreviations: ADA; American Diabetes Association ISPAD; International Society of Pediatric and Adolescent Diabetes.

Keywords: Diabetes, Exercise, Children

\section{The phenotypic variability in tetrahydrobiopterin deficiency, an Iranian experience}

Zaman T, Einollahi N, Moradian R

Tehran University of Medical Sciences \& Iranian National Society OF SSIEM, Iran

Background: Tetrahydrobiopterin (BH4) activates phenylalanine hydroxylase as well as tyrosine 3 hydrxyolase and tryptophan hydroxylase. BH4 deficiency causes biogenic amine synthesis defect and none specific neurologic abnormalities as well. BH4 deficiency, a mild form of HPA is mostly accompanied by a deficiency of the neurotransmitters such as dopamine and serotonine, so causes severe cases if not diagnosed. Elevated urine neopterin as well as hyperphenylalaninemia is most often in favor of diagnosis of 6-pruvoyl-tetrahydropterin synthase (PTPS)deficiency. Thus far, over 250 patients with PTPS were described with various degrees of phenotypic severity. Treatment with BH4, levodopa, 5HTP and diet low in phenylalanine, if it is high, is effective in the most of them. Dopamine agonist(pramipexol) may well result in faciliated clinical response to L-dopa therapy in BH4 deficiency. The Objective of the study was to study clinical, laboratory data, and outcome of patients with BH4 deficiency.

Methods: We studied 14 patients suspicious to have biogenic amine synthesis defect, among 257 patients of PKU group and 534 patients with non-specific neurologic manifestations without defined diagnoses.

Findings: 14 cases; ( 4 female, 10 male), age range: $26 \mathrm{mo}-$ 27yr, onset; adulthood(3), juvenile(3). Clinical findings were mild psychomotor retardation in $14(14 / 14)$, convulsion in $5(5 / 14)$, hypersalivation in $5(5 / 14)$, distal chorea 2 (2/14), temperature disturbances in $3(3 / 14)$ autism 1 (1/14), oculogyric crises; 1, awkward walking 4, hypertonia (3) hypotonia (1) hyper/hypo (1). High urine neopterin and urine neopterin/creatinine in 14(14/14), high plasma phenylalanine; 3(3/10) and low urine VMA;6(6/10). Treatment with levodopa-carbidopa,5 hydroxy tryptophan and BH4 was effective in those which was started in early stages of symptomatic disease; onset; adulthood (3) juvenile (3), and childhood (3).

Conclusion: In any undiagnosed neurologic disease we should consider biogenic amines synthesis defects and recommend urine, CSF investigations to treat as early as possible to prevent brain damage and to improve outcome. Each new case of PKU should be tested for malignant types to be treated soon. Mild forms of PTPS deficiency can cause movement disorders in adults. Two adult cases were reported in this paper.

Keywords: Hyperphenylalaninemia, Malignant PKU, Biogenic Amine Synthesis Defect

\section{Association of 25-hydroxy vitamin D levels with indexes of generalized and abdominal obesity in Iranian adolescents: the CASPIAN-III Study}

Jari M, Kelishadi R, Qorbani M, Moafi M, Esmaeil Motlagh M, Keikha M, Ardalan G

Isfahan University of Medical Sciences, Iran

Background: This study aims to determine the associationof serum 25-Hydroxy vitamin D (25(OH)D) levels with measures of generalized and abdominal obesity in Iranian adolescents.

Methods: This nationwide study was conducted among 1090 students, aged 10-18 years, living in 27 provinces in Iran. Serum concentration of $25(\mathrm{OH}) \mathrm{D}$ was analyzed quantitatively by direct competitive immunoassay chemiluminescence method. Body mass index (BMI) and waist-to-height ratio (WHtR) were considered as measures of generalized and abdominal obesity, respectively.

Findings: The mean age of participants was $14.7 \pm 2.6$ years; mean BMI $19.3 \pm 4.2 \mathrm{~kg} / \mathrm{m} 2$ ), the median serum $25(\mathrm{OH}) \mathrm{D}$ was $13.0 \mathrm{ng} / \mathrm{mL}$ (interquartile range $6.8-27.4)$. A total of $40 \%$ were vitamin D deficient and $39 \%$ were vitamin D insufficient. Serum $25(\mathrm{OH}) \mathrm{D}$ level was not associated with BMI and WHtR.

Conclusion: We did not document significant association between serum 25(OH) D level and anthropometric measures. This finding may be because of considerably high prevalence of hypovitaminosis D in the study population. 
Keywords: Obesity, 25hydroxy Vitamin D, Adolescents

\section{PKU: treatment standards in Europe}

\section{Iwona Wojcicka B \\ Poland}

The ESPKU is publishing this PKU:Closing the Gaps in Care report with one clear objective -to give PKU patients and their families a greater voice. The ESPKU envisages to initiate discussions and actions to enable a fairer deal for all PKU patients no matter where they live.

The research and this report do not amount to scientific review of the management of PKU in Europe. The research on which this report is based to provide a useful insight into some of the issues affecting people with PKU and their families and gives an indication of some of the discrepancies in PKU care between some European countries. Phenylketonuria (PKU) is caused by a deficiency of phenylalanine hydroxylase, the enzyme converting the amino acid phenylalanine (Phe) into tyrosine. This causes the level of Phe in the blood to rise. Cases vary from mild to severe, with severe cases having very high blood Phe concentrations. A severe case, left untreated, will result in profound and irreversible mental disability. An abnormally high blood level of Phe is known as hyperphenylalanineaemia (HPA). This may be caused either by PKU or by a deficiency in one of the enzymes synthesizing or recycling the co-factor tetrahydrobiopterin (BH4), which stimulates the above called enzyme phenylalanine hydroxylase. Almost all European countries carry out neonatal screening programs that identify HPA cases at birth. Cases with HPA are referred to special clinics to identify whether the child has PKU or BH4 deficiency. Once identified, a child with PKU is placed on a low Phe diet which, if initiated soon after birth, will prevent most of the neurological complications. In practice, a low Phe diet consists of a diet low in natural protein combined with a high intake of a protein substitute which has all amino acids but Phe. Due to the restrictive nature of this diet, however, compliance tends to diminish as the child gets older. Drug treatment can allow some PKU patients to keep their Phe levels under control while on a less restrictive diet. However, data on long term drug treatment compliance on large scale of patient population is further needed.

\section{Diagnose communication in Inborn Error of Metabolism}

\section{Iwona Wojcicka B \\ Poland}

Giving a diagnosis of an inborn error of metabolism is one of the most important moments for parents and patients with a significant laboratory result (after newborn screening or clinical presentation) as well as for the metabolic specialist. The given information will change the life of the family in a meaningful way. Parents and patients will remember the situation for the rest of their life. Likewise it will be the start of a close relation between the family and the metabolic team. The material of this modular system aims to support knowledge, communication and cooperation. It has been developed by specialists in the field with long-standing experience of treatment, care and research of inborn errors of metabolism. It has been repeatedly tested and modified and is based on the evidence that each patient asks the same questions: 1. What does the disorder mean? (terminology), 2. How was it discovered? (diagnostics), 3. How did I get it? (etiology), 4. What exactly is wrong? (nosology), 5. how does it show? (symptomatology), 6. Is it severe, dangerous, perilous, life-shortening? (outcome), 7. What can I expect? (prognosis), 8. What can be done (how, how often, how long, by whom)? (treatment/cure), 9. Will it recur? (development \& transmission)

\section{Case presentation about non ketotic hyper glycinemia}

\section{Dalili $S$}

17 Shahrivar Hospital, Rasht, Iran

You are called to the newborn nursery regarding an 8 hour old female infant who is listless and not interested in feeding. On exam, the baby is severely hypotonic and lethargic but no other obvious abnormalities are noted. Glucometry shows normal glucose. Blood gas, complete metabolic profile, CBC, plasma ammonia, lactate and urinalysis all show normal results. Chest X-ray comes back normal. Along with other possibilities, you suspect a neuromuscular disorder and consult neurology. Maintenance IVFs are started. Pregnancy history is significant for decreased fetal movements. While awaiting neurology consult, the baby has apnea spells and develops myoclonic jerks. and is intubated. An EEG is performed and shows a "burst suppression" pattern.

Keywords: Non Ketotic, Hyperglycinemia, Convulsion

\section{Is it not the time to do something for vitamin D deficiency?}

\section{Kosaryan M, Zafari M, Aliasgharian A, Mosawi M, Hadian A \\ Mazandaran University of Medical Sciences, Iran}

Background: Vitamin D deficiency (insufficiency) is a common nutritional disorder in our country. Vitamin D is important for the health of all human beings since birth. This study has been conducted in order to summarize epidemiologic researches regarding vitamin D deficiency in different parts of the country and to conclude if food fortification is necessary. Study designed in Thalassemia Research Center, Sari, Iran.

Methods: This was a narrative review about the current situation of vitamin D deficiency in Iran in December 2013. Related literature in Persian and English were explored since 10 years ago. Data source of this study was Medline, SID, PubMed, Scopus, Request, Web of knowledge, Springer, Ovid, Google scholar.

Findings: We found 27 cross-sectional researches regarding vitamin D status in Iran from 2003-2013. Also, there was a Meta analysis that has been done in 2008 . $25(\mathrm{OH}) \mathrm{D} 3$ was measured mostly by radio immune assay (RIA) method. Most studies were done on adults. Definition of vitamin D deficiency was based on cut off of the kit in most studies; however, in some the serum parathyroid hormone (PTH) was measured and cut off for diagnosis was based on increased PTH. Maximum rate of severe vitamin D deficiency was reported as 47\% in 2011. 
Conclusion: Vitamin D deficiency is very frequent in Iran. Dealing with a national important nutritional problem is important. Fortification of a suitable food or edible product which in this case would be milk or cooking oil is one way. Increase public awareness about the problem and motivate people to do something in their expense is another option. In this case, taking vitamin D supplements as a regular basis daily, weekly or any other routines which could be available and cost effective may solve the problem. For all above one year old persons, our recommendation is to take $300,000 \mathrm{IU}$ (as IM injection or oral dose) of vitamin D as a starting dose, then take 50,000 IU oral dose (as one pearl) every 3 months.

Keywords: Vitamin D2, Vitamin D3, Osteomalacia, Rickets, Prevention, Treatment, Fortification, Iodine, Iron

\section{The influence of late treatment on oral communication skills in Farsi speaking children with Phenylketonuria}

\section{Soleymani Z, Keramati N, Rouhani F, Jalaei S Tehran University of Medical Sciences}

Background: Screening programs have commenced for Iranian children with Phenylketonuria (PKU) in recent years. The aim of the study was to investigate oral communication skills in late treated children with PKU to find the influence of treatment onset on these skills. Methods: In this cross-sectional study, 22 late-treated PKU and 8 early-treated PKU aged 4-6.5 evaluated by: a) Wechsler preschool and primary scale of intelligence (WPPSI) consist of verbal intelligence quotient (VIQ) and total intelligence quotient (TIQ) and by b) test of language development-third edition (TOLD-p:3) containing composite quotients of spoken language, listening, speaking, semantics, syntax, and organizing. Dependent on treatment onset, PKU children are divided into 3 groups: early-treated PKU $(n=8)$ (patient from newborn screening program), late-treated PKU diagnosed before one year old $(\mathrm{n}=9)$, and late-treated PKU diagnosed after one year old $(n=13)$. For all patients time of diagnosis and treatment onset was the same. The oral communication skills matched within PKU children and matched between PKU children and normal control. The relation between blood Phe level with spoken language and VIQ also computed. Findings: Normal groups performed significantly better than early-treated children in all composite quotients of TOLD-p: 3 and VIQ (p<0.002). Early-treated PKU children significantly performed better than late-treated children in spoken language $(\mathrm{P}<0.01)$, speaking $(\mathrm{p}<0.04)$, syntax $(\mathrm{p}<0.02)$ and VIQ $(\mathrm{p}<0.002)$. There were negative relation between Phe and VIQ ( $\mathrm{r}=-0.79)$ in early-treated and negative relation between Phe and spoken language $(\mathrm{r}=-$ $0.71)$, organizing $(r=-0.82)$, and semantics $(r=-0.82)$ in latetreated PKU children that had been detected before one year old.

Conclusion: We discovered that postponement in diagnosis and treatment onset affects children with PKU suffer more oral communication impairment. The sooner therapy is initiated; the more recovery in oral communication skills is obtained so that late-treated children before one year old compared to after it had superior performance in oral communication and cognitive function.

Keywords: Phenylalanine, treatment onset, oral communication

\section{Association of serum 25-hydroxy vitamin D levels and liver enzymes in a nationally representative sample of Iranian adolescents: the CASPIAN III Study}

\section{Kelishadi R, Heshmat R, Motlagh ME, Ardalan G Isfahan University of Medical Sciences}

Background: Hypovitaminosis D is highly prevalent, and has several adverse health effects. This study aimed to assess the relationship of serum concentrations of 25hydroxy vitamin $\mathrm{D}[25(\mathrm{OH}) \mathrm{D}]$ and liver enzymes in adolescents.

Methods: This population-based cross-sectional survey was conducted among a nationally representative multistage sample of 1095 adolescents (52\% boys), aged 10-18 years, living in different provinces of Iran. Serum 25(OH)D concentration $<30 \mathrm{ng} / \mathrm{mL}$ was considered as hypovitaminosis D, and liver enzymes (alanine aminotransaminase, ALT and aspartate aminotransaminase, AST) of > $40 \mathrm{U} / \mathrm{L}$ as high level. To determine the association between serum $25(\mathrm{OH}) \mathrm{D}$ categories and elevated levels of liver enzymes, multiple regression models and linear regression analysis were applied, after adjustment for potential confounders. Odds ratio (OR) 95\% confidence interval $(\mathrm{CI})$ of serum $25(\mathrm{OH}) \mathrm{D}$ and elevated liver enzymes were assessed by logistic regression analysis. Findings: Higher rates of vitamin D deficiency were documented among individuals with increased levels of liver enzymes. Compared to boys, median of $25(\mathrm{OH}) \mathrm{D}$ was lower in girls with elevated levels of liver function tests (12.75 vs. $25.60 \mathrm{ng} / \mathrm{mL}$ for ALT, and 13 vs. $14.10 \mathrm{ng} / \mathrm{mL}$ for AST), with marginally significant gender differences regarding AST.

Conclusion: We found a relatively high frequency of hypovitaminosis D among adolescents with abnormal liver function. Further prospective studies are needed to examine these associations from early life.

Keywords: Vitamin D, liver function tests, adolescents

\section{Dental problems in hypophosphatemic rickets}

\section{Rahmani P, Rabbani A, Ziaee V, Ghodoosi SH} Children's Medical Center, Tehran University of Medical Sciences, Tehran, Iran

Background: Hypophosphatemic Rickets is an uncommon metabolic bone disorder which affects all ages and both sex. It is characterized by low concenteration of serum levels, impairement of mineralization of bone matrix and teeth with variable etiology. Dental problems have not been described well in previous studies.

Methods: All hypophosphatemic rickets patients who came to referral clinic during 2008-2010 enrolled in this study. All patients had low phosphorous leveland high ALP \& normal PTH \& 25-hydroxy vit D and normal or low serum calcium. After diagnosis by a all patients were examined by dentist for enamel hypoplasia, taurodontism, dental abcesses, dental caries and dentition delay.

Findings: Ninteen patients were enrolled in this study. The average age of patients was 10 years (range 3-17). 79\% patients had regular follow up after diagnosis of background disease. Dental caries and delay in dentition were most prevalent each one $(47.7 \%)$ followed by enamel hypoplasiahn in $42.1 \%$ patients. Other problems were 
taurdontism in $15.8 \%$ patients, dental abcess and gingivitis in $10.9 \%$.

Conclusion: Hypophosphatemic rickets is a disease with different clinical features that one of them is dentalblem. Dental caries is the most common problem.

Keywords: Dental Caries, Hypophatemia, Rickets, Enamel Hypoplasia

\section{Approach to hypocalcemia with three presentation}

\section{Koohmnanaie SH}

Gillan University of Medical Sciences, Iran

Case Presentation: Case 1: A 2-year-old girl was referred to our hospital because of failure to walk and absent teeth development. The patient appeared to be well nourished and content. His body mass index, height and weight were $20.1 \mathrm{~kg} / \mathrm{m} 2$ (90th percentile), $87 \mathrm{~cm}$ long (25th percentile) and $14 \mathrm{~kg}$ (75th percentile) respectively. Palpation of the patient's extremities revealed prominent, widening of wrist and ankle. The result of a total serum calcium test was 7 $\mathrm{mg} / \mathrm{dl}$. Case 2: A 7-year-old boy had short stature $(<3$ rd percentile) which presented with trunkal hard, nodular skin lesions, and he had mild obesity and developmental delays. Because a skin biopsy demonstrated subcutaneous calcification, his total serum calcium level was measured and found to be $7.5 \mathrm{mg} / \mathrm{dl}$. Case 3: A 14-year-old girl presented with intermittent numbness of her extremities, and "lost control" of her right leg and falling. A CT scan showed the basal ganglia calcification. Her total serum calcium and phosphorus level was 6 and $7 \mathrm{mg} / \mathrm{dl}$ respectively.

Keywords: Hypocalcemia, Calcium, Short stature 